\title{
P01-294
}

\section{DEPRESSION IN CORONARY HEART DISEASE}

A. Stetkiewicz ${ }^{1}$, A. Goch ${ }^{2}$, A. Borkowska ${ }^{1}$

${ }^{1}$ Department of Medical Psychology, Medical University, ${ }^{2}$ Department of Cardiology, Medical University, 1 st Chair of Cardiology and Cardiac Surgery, Lodz, Poland

Background and aims: Current studies showed a significant association between depression and Coronary Heart Disease (CHD) Depression is a significant etiopathogenetic factor of CHD and caused with worsening of the course of the illness. The aim of this study was to asses the relationship between the intensity of depressed symptoms and the course of the illness and also demographic factors.

Methods: The group of $111 \mathrm{CHD}$ patients (89 male, 22 female) aged 41-65 years (mean 55 $\pm 5,6$ ). The individuals were assessed according to the following criteria: sex, job status (working, unemployed, retired), vascular diseases in family, myocardial infarction (MI) in history, number of MI, patients with acute MI were divided according to the duration of time from the onset of $\mathrm{MI}$ pain (patients who passed Ml within a week and above a week from the onset of $\mathrm{MI}$ ). The intensity of depressive symptoms were assessed by subjective Beck Depression Inventory (BDI) and objective Hamilton Depression Rating Scale (HDRS).

Results: The intensity of depressed symptoms in BDI was 9,9 $\pm 7,5$ and in HDRS 6,6 $\pm 4,5$. Females showed significant higher level of depression compared with males. In patients who passed MI within a week before the evaluation as compared with those whose term after MI was longer than a week the less level of depression was observed. Also active workers presented significantly less degree of depressive symptoms than pensioners.

Conclusion: The results obtained showed the association between the intensity of depression and the more severe course of the CHD, especially in female and pension patients.

Acknowledgements: This research was supported by grant 502-16-653 Medical University of Lodz 